\title{
Self-consistent approach for spectral properties of single alkali adatoms on $\mathrm{Cu}(111)$
}

\author{
S. Achilli, ${ }^{1}$ M. I. Trioni, ${ }^{2,3}$ and E. V. Chulkov ${ }^{3,4}$ \\ ${ }^{1}$ Dipartimento di Scienza dei Materiali, Università di Milano-Bicocca, via Cozzi 53, I-20125 Milano, Italy \\ ${ }^{2}$ CNR, National Research Council of Italy, ISTM, Via Golgi 19, I-20133 Milano, Italy \\ ${ }^{3}$ Donostia International Physics Center (DIPC), Paseo Manuel de Lardizabal 4, E-20018 San Sebastian, Spain \\ ${ }^{4}$ Departamento de Física de Materiales and Centro de Física de Materiales (CFM)-Materials Physics Center (MPC), E-20018 San \\ Sebastian/Donostia, Spain and Facultad de Ciencias Químicas, UPV/EHU, Apdo. 1072, \\ E-20080 San Sebastián/Donostia, Basque Country, Spain \\ (Received 17 October 2011; published 6 January 2012)
}

\begin{abstract}
We investigate the nature of the adatom-surface interaction in the adsorption of alkali atoms on $\mathrm{Cu}(111)$. Our calculation, exploiting the embedding approach in a density functional theory framework, describes an isolated atom on a metallic surface, which is modeled via a one-dimensional modulated potential. The absence of empirical term in the Hamiltonian guaranties a completely ab initio determination of the electronic properties of the system. The role of the projected energy gap in determining lifetime and binding energy of the adatom resonances is evidenced. On the basis of the electronic properties of the adsorbed alkali atoms the covalent/ionic nature of the bonding with the surface is analyzed.
\end{abstract}

\section{INTRODUCTION}

The adsorption of alkali atoms on metal surfaces has been investigated for decades. The main peculiarity of such a system, i.e., the reduction of the substrate work function due to the surface dipole, ${ }^{1-7}$ was exploited for practice applications in technology: The enhancement of electron and ion emission favored the development of high-electron-emission cathodes and thermionic converters, while the promotion of heterogeneous reactions allowed us to achieve a superior selectivity and reactivity in catalysis.

Despite the apparent simplicity of the system that counts only one valence electron at disposal for the adatom-substrate interaction, the theoretical characterization of the electronic properties of the system is not straightforward and the nature of the chemical bonding between the atom and the surface was the object of a long debate. ${ }^{8-12}$

Since the pioneering work of Langmuir the picture of an ionized alkali atom, whereby it donates its valence electron to the metal substrate, has been hypothesized. ${ }^{13,14}$ Such an interpretation was revised in the quantum-mechanical treatment of Gurney. He pointed out that a partial ionization of the adatom occurs due to the broadening of the alkali-metal $s$ valence level that results in a partially filled resonance. ${ }^{15}$ This picture also provided the basis to explain the coverage-dependent behavior of the work function that is related to the surface dipole. The lowering of the work function with increasing coverage and its stabilization for the full monolayer was attributed to the depolarization field induced by the other adatoms, leading to the progressive filling of the resonance.

A series of simplified models, ${ }^{16-18}$ also based on atomistic theories, ${ }^{19,20}$ were hypothesized in following years, supporting the Gurney explanation. However, only with the advent of the density functional theory (DFT ${ }^{21}$ were $a b$ initio methods exploited for a theoretical characterization of the system. ${ }^{10,22,23}$

These methods can essentially be divided into those that account for a system of a finite size and the extended substrate ones. In the former type, the limited extension of a cluster or a thin $\operatorname{slab}^{24,25}$ prevents the description of the effects related to the hybridization between alkali atomic states and the continuous band of the metal. In the latter class, the more complex approach often required an oversimplified description of the substrate: The methods able to account for a really isolated adatom were mainly based on a jellium description of the surface. ${ }^{26-28}$ Differently, a realistic potential for the surface was adopted using a dilute overlayer for the adsorbate instead of a really isolated adatom. ${ }^{29}$ The adatom-adatom interaction leads in this case to the formation of electronic bands in the overlayer. ${ }^{30,31}$

It is noteworthy that the methods exploiting a semi-infinite substrate approach can directly obtain the elastic contribution to lifetime, because they account for the hybridization of the atomic levels and substrate states. Supercell approaches give the linewidth as the envelope of the discrete states forming the resonance. $^{32}$

The electronic spectrum of alkali adatoms on jellium surfaces, both in the Dyson approach ${ }^{33,34}$ and the embedding treatment, ${ }^{28}$ showed wide resonances, partially filled. The large hybridization with substrate states and the energy position of the main resonance with respect to the Fermi level lead to the conclusion that the adatom-substrate bond is partially covalent, also at low coverages. The finite-size approaches, neglecting the hybridization of the adatom-metal electronic states, attributed an ionic character to the adatomsurface interaction. The experiments show that the realistic electronic properties differ substantially from those obtained on jellium, revealing unoccupied and extremely narrow atomic resonances. ${ }^{35-43}$

Recently, a phenomenological model has shown that for a good description of the electronic properties of the alkali adatom systems is crucial to account for the main characteristics of the electronic properties of the substrate. ${ }^{44,45}$ Nevertheless, such a model is also based on the assumption of a complete ionization of the adatom.

Here we propose a method that combines the advantages just discussed of a really isolated adatom on a semi-infinite substrate and a self-consistent procedure that gives an $a b$ initio description of the atom-surface interaction. We exploit 
the embedding method, already applied to treat surfaces and thin films ${ }^{46-48}$ and single atoms on jellium surfaces. ${ }^{49}$ Our improvement with respect to calculations on jellium is the use of a modulated one-dimensional pseudopotential for the substrate. ${ }^{50}$

We are aware that such a potential is not as accurate as three-dimensional self-consistent density functional potentials that accurately include the band structure of the underlying bulk metal. Nevertheless, despite its simplicity, the potential adopted accounts for the main electronic properties of the substrate (energy gap and surface states) that play an active role in the adatom-surface interaction. In this sense we believe that it can give an interesting insight into the physical aspects that affect the adatom induced resonances.

The paper is organized as follows: in Sec. II a basic description of the method is given. Sec. III is devoted to the analysis of the electronic properties of the interacting system. In Sec. III some peculiar aspects are discussed in order to clarify the nature of the adatom-surface interaction. Finally, in Sec. V, the conclusions are given.

\section{METHOD}

The major difficulty in the theoretical description of single adatom adsorption is the lack of periodicity. It has been clear from the first results that some benefit could be obtained if the spatial region proper of the atom is distinguished in the calculation from the unperturbed extended substrate. ${ }^{22,33,34,51}$

The embedding method was developed in the wake of such idea. In this Green's function-based approach the effective Schrödinger equation is solved in a limited volume, which includes the perturbation induced by the impurity. The inclusion of an embedding potential in the Hamiltonian furnishes the correct boundary conditions for matching with the unperturbed solution outside the embedded region. ${ }^{49,52}$

The preliminary step for the forthcoming treatment of an isolated adatom adsorbed on a surface is the calculation for the clean surface. We model the $\mathrm{Cu}(111)$ surface using a pseudopotential dependent only on $z$, the coordinate normal to the surface.$^{50}$ Although the corrugation parallel to the surface is neglected, the potential is suitable to model the adatom-surface interaction. Indeed, due to the absence of periodicity parallel to the surface, an integrated contribution on the whole pristine surface Brillouin zone has to be considered in the subsequent adsorption problem. Furthermore, in such an approach the $d$ band of copper, which lies from -4 to $-1.5 \mathrm{eV}$ with respect to the Fermi level, is completely neglected. However, due to the strong localization of the $d$ states and their energy position, the lack of the $d$ band does not affect the adatom-induced electronic states that lie at higher energies, as will be shown in the following. For the clean surface calculation we use an embedded slab containing three atomic layers of the substrate and $20 \mathrm{a}_{0}$ (Bohr) of vacuum. Details regarding the construction of the embedding potential for such a configuration are given elsewhere..$^{53}$

In view to tackle the problem of a single adatom on a surface, we use a spherical embedded region to host the adatom. Once the Green's function of the clean surface has been calculated, the new embedding potential on the spherical boundary can be obtained following the method already
TABLE I. Equilibrium adsorption distances $(d)$, calculated energies (with respect to $\left.E_{F}\right)$ and linewidths $\left(\Gamma_{\mathrm{el}}\right)$ of the unoccupied resonances of all the alkali/ $\mathrm{Cu}(111)$.

\begin{tabular}{ccccccc}
\hline \hline & & $\mathrm{Li}$ & $\mathrm{Na}$ & $\mathrm{K}$ & $\mathrm{Rb}$ & $\mathrm{Cs}$ \\
& $d\left(a_{0}\right)$ & 4.0 & 4.69 & 5.49 & 5.75 & 6.0 \\
\hline$\sigma$ & $\mathrm{E}(\mathrm{eV})$ & 2.94 & 2.62 & 2.67 & 2.62 & 2.74 \\
& $\Gamma_{\mathrm{el}}(\mathrm{meV})$ & 38 & 114 & 48 & 30 & 22 \\
$\pi$ & $\mathrm{E}(\mathrm{eV})$ & 4.36 & 4.17 & 4.0 & 3.93 & 3.8 \\
& $\Gamma_{\mathrm{el}}(\mathrm{meV})$ & 920 & 98 & 28 & 36 & 284 \\
$\sigma^{\prime}$ & $\mathrm{E}(\mathrm{eV})$ & - & - & 4.43 & 4.28 & 3.9 \\
& $\Gamma_{\mathrm{el}}(\mathrm{meV})$ & - & - & 270 & 384 & 850 \\
$\delta$ & $\mathrm{E}(\mathrm{eV})$ & - & - & 4.85 & 4.61 & 3.98 \\
& $\Gamma_{\mathrm{el}}(\mathrm{meV})$ & - & - & 872 & 714 & 604 \\
\hline \hline
\end{tabular}

reported in Ref. 49. In this method the only requirement is the invariance of the clean surface potential under translation along the surface. This is indeed satisfied by the adopted onedimensional model potential. The last ingredient to perform a DFT ab initio calculation inside the embedded sphere is the ionic potential due to the positive charge of the clean surface. This ionic term is obtained subtracting the Hartree and exchange-correlation contributions to the model potential used for the clean surface calculation. ${ }^{50}$ Such a term, added to the spherical nuclear one, acts as the external potential of the Kohn and Sham equation inside the sphere.

The calculation for alkali adatoms on $\mathrm{Cu}(111)$ is performed in a large embedded sphere, with a radius of $12 \mathrm{a}_{0}$. This choice is motivated by the expected charge transfer in the alkali-surface interaction that could perturb the potential in a large volume. The cutoff for the basis set has been chosen consistently, taking spherical waves up to $l=18$ while the kinetic energy cutoff is 7.5 Ry. Muffin tins have a radius of $4.4 \mathrm{a}_{0}$. The equilibrium distances (see Table I) are obtained by use of the DFT calculation for a very dilute overlayer structure in order to make negligible the lateral adatom-adatom interaction. ${ }^{43}$

\section{RESULTS}

In this section we present the calculated ground-state electronic properties of the adatom-surface interacting system.

In the absence of the adatom the density of states (DOS) of the clean surface appears as depicted in the upper panel of Fig. 1 (red solid line). The DOS is evaluated in a sphere of radius of $5 \mathrm{a}_{0}$ whose center is at $6 \mathrm{a}_{0}$ from the surface atomic layer. Such a quantity is obtained through an integration in $k_{\|}$that can be performed considering the DOS of $\mathrm{Cu}(111)$ at $\bar{\Gamma}$ (lower panel of Fig. 1) and the parabolic dispersion of the electronic states. In the range of the surface-projected energy gap at $\bar{\Gamma}$, i.e., between -0.89 and $4.25 \mathrm{eV}$, the DOS displays a constant value. The two steps at -0.39 and $4.12 \mathrm{eV}$ correspond to discrete surface states: The feature below the Fermi level is related to the Shockley surface state (SS) while the second is due to the $n=1$ image potential state. The binding energy of the latter is close to the upper edge of the symmetry gap and it appears merged with the upper bulk band edge, due to the smoothening of the DOS behavior by the inclusion of a small imaginary part in the energy. 


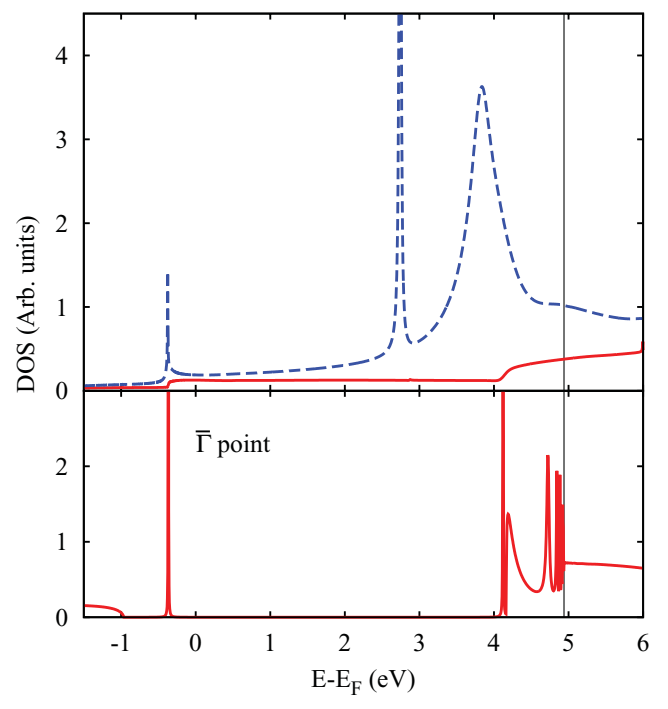

FIG. 1. (Color online) (Lower panel) DOS of $\mathrm{Cu}(111)$ at the $\bar{\Gamma}$ point. (Upper panel) $k_{\|}$-integrated DOS of $\mathrm{Cu}(111)$ (red solid line) and DOS of $\mathrm{Cs} / \mathrm{Cu}(111)$ (blue dashed line) evaluated in a spherical volume of radius of $5 a_{0}$. Energy is given with respect to the Fermi level $\left(E_{F}\right)$, while the vacuum level is marked by a vertical line.

The $k_{\|}$-integrated DOS of $\mathrm{Cu}(111)$ is the quantity to be compared with the DOS of the interacting system. In fact, due to the lack of translational invariance in the presence of the adatom, the $k_{\|}$resolved DOS has no more physical meaning and all the $k_{\|}$components will contribute to the DOS at a certain energy.

On the adsorption of a single adatom, the DOS is partially modified, accounting for some new features. The blue dashed line in Fig. 1 represents the local DOS (LDOS) of $\mathrm{Cs} / \mathrm{Cu}(111)$, which we take as representative system (all the alkali adatoms induce similar electronic properties, as can be seen in Fig. 2). The DOS is evaluated in the spherical atomic region of radius of $5 \mathrm{a}_{0}$. Below the Fermi level a sharp peak is visible near the Shockley surface state edge. This peak can be attributed to a bound state due to the localization of the Shockley surface state, as confirmed also by its symmetry: Both the peak and the pristine SS have $m=0$ character, where $m$ is the azimuthal quantum number.

As shown by Simon,,${ }^{54}$ an attractive perturbation acting on a two-dimensional electron gas always originates a bound state. In the present case the attractive potential is due to the alkali adatom while the surface state, trapped between the vacuum potential barrier and the energy gap of the substrate, can be viewed as a quasi-two-dimensional electron gas. Differing from jellium, the modulated potential used in our approach allows us to reproduce the discrete Shockley state in the energy gap and, thus, to predict such a localization.

The adatom-induced split off of SS has been observed experimentally on $\mathrm{Cu}(111)$, due to the adsorption of $\mathrm{Cu}$ atoms and magnetic impurities. Scanning tunneling spectroscopy (STS) experiments ${ }^{55-57}$ estimated an energy localization for these systems of the order of tens of $\mathrm{meV}$, as confirmed also by theoretical calculations. ${ }^{57,58}$ The only theoretical prediction of the split off due to alkali atom adsorption was

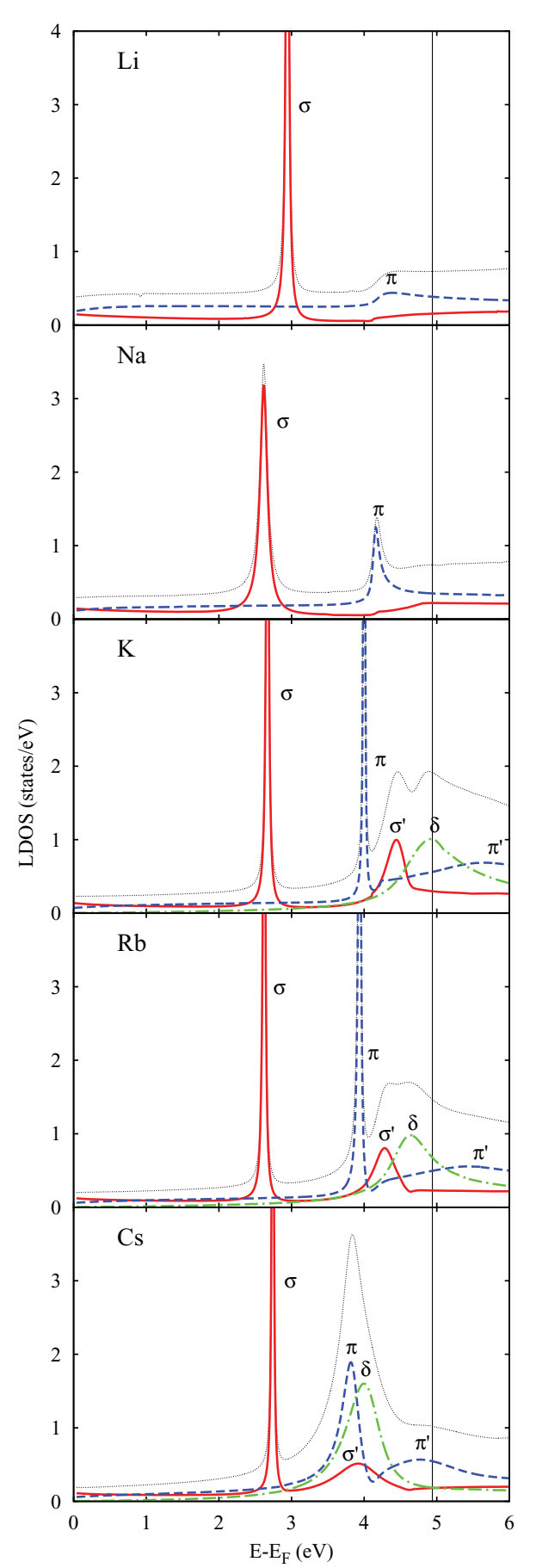

FIG. 2. (Color online) Unoccupied LDOS of alkali on $\mathrm{Cu}(111)$ at the equilibrium adsorption distance (dashed line). Red solid, blue dashed, and green dotted-dashed lines show $m=0, m=1$, and $m=2$ contributions, respectively.

furnished by Gauyacq et al. ${ }^{59}$ who used the wave-packet propagation (WPP) method, obtaining for $\mathrm{Cs} / \mathrm{Cu}(111)$ an energy localization of the SS of a few meV. Our results are in agreement with this value: We find a similar localization 
for all the heavy alkali adatoms ( $\mathrm{K}, \mathrm{Rb}$, and $\mathrm{Cs})$, while it is larger for $\mathrm{Li}$ and $\mathrm{Na}$ (see the next section). The corresponding peak in the DOS of $\mathrm{Cs} / \mathrm{Cu}(111)$ is characterized by a small linewidth, which is due to the elastic broadening caused by the hybridization with substrate states. ${ }^{31}$ Although this state lies in the surface projected energy gap at $\bar{\Gamma}$, the absence of translational invariance at the surface allows hybridization with states at different $k_{\|}$.

The unoccupied DOS shows a general increase of the number of electronic states in the whole energy range and the appearance of two new peaks that are related to the electronic energy levels of alkali atom. Regarding their assignment, many studies were devoted in the past to the interpretation of the features observed above the Fermi level.

The experiments, performed using various spectroscopic techniques able to accede to excited states [e.g., inverse photoemission, two photon photoemission (2PPE), electron energy loss spectroscopy, etc.], agree in identifying a resonance above the Fermi level as due to the adsorbate electronic state. ${ }^{36,37,40,60,61}$ The atomic character of such a feature is confirmed by its coverage-dependent binding energy ${ }^{35}$ and by its dispersion-less behavior in $k_{\|}$for very low coverages. ${ }^{36}$ Due to its symmetry, i.e., its largest intensity for emission normal to the surface, this resonance has been identified as $\sigma$ $(m=0) .{ }^{39}$ Recent 2PPE experiments have shown that for all the alkali/Cu(111) systems the binding energy of this state is about $2 \mathrm{eV}$ from the vacuum level $[\mathrm{Cu}(111)$ work function $=$ $4.94 \mathrm{eV}]$, in the limit of zero coverage. ${ }^{43}$

Furthermore, time-resolved two-photon photoemission experiments that give an accurate description of the decay dynamics of excited states revealed another striking property of this resonance: an extremely long lifetime, of the order of few tens of femtoseconds at low coverages. ${ }^{40-42}$ A long decay time is relevant for physical processes exploiting the alkali resonance as transient state, for example, increasing the efficiency of photo-induced desorption processes. ${ }^{62,63}$ Angleand time-resolved photoemission experiments (AR-2PPE, TR-2PPE) characterized also a second resonance, at higher energies with respect to the $\sigma$ one, which displays a maximum in intensity off-normal and has been thus identified as a $\pi$ resonance $(m=1){ }^{64}$

For what concerns the theoretical studies, different approaches can be found in the literature. Early calculations on jellium surfaces gave for $\sigma$ a binding energy near the Fermi level and a width of $1 \mathrm{eV} \cdot{ }^{18,22,28}$ More recently, Zhao et $\mathrm{al}^{43}$ used a semiempirical potential that includes the one-dimensional modulated potential for the substrate and a pseudopotential for the adatom and takes into account the Coulomb interaction between the image charges. This modeling of the system is able to reproduce the binding energy of the $\sigma$ resonance and also explains the period independent behavior as due to the compensation between the ionization potential of the alkali and the image charge repulsion. ${ }^{44}$ A similar potential, built assuming an ionized adatoms in front of the surface, was exploited by Borisov et al. ${ }^{65}$ to demonstrate the key role of the surface projected substrate band gap on the lifetime of the alkali-induced resonance. Indeed, the resonant charge transfer (RCT) of electrons from the adatom to the surface becomes attenuated when an energy gap in the surface band structure prevents the tunneling of electrons in the direction normal to the surface. Niedfeldt et $a l .{ }^{32}$ have performed a slab calculation for a very dilute overlayer of $\mathrm{Li}$ on $\mathrm{Cu}(111)$. They extract the elastic width of the $\sigma$ resonance, through a DFT-based deconvolution scheme, from the envelope of the discrete states forming the peak. ${ }^{66}$

The LDOS obtained by using our method displays a narrow resonance at $2.7 \mathrm{eV}$ from the Fermi level. This peak has $m=0$ character and can be identified with the $\sigma$ resonance found experimentally. ${ }^{36,37,40,43}$ Many interpretations were given in the past regarding the assignment of this peak to a parent atomic level. ${ }^{22,37,38,61}$ Nevertheless, a characterization in term of the $l$ quantum number would not be appropriate because $l$ has no meaning at the surface due to the broken rotational symmetry. The $\sigma$ resonance is definitely related to the $s$ valence level of the alkali adatom, although it acquires also a $p_{z}$ character due to the mixing of the $m=0$ components in the cylindrical field at the surface. On the other hand, the $m=0$ contribution that is mainly related to the parent $p_{z}$ atomic level is delocalized at higher energies, in correspondence of the upper bulk band. Our $a b$ initio binding energies for the $\sigma$ resonance of alkali adatoms on $\mathrm{Cu}(111)$ are in agreement with those reported by Zhao et al. ${ }^{43}$ and display the previously discussed period-independent behavior at the equilibrium distance. Near the surface we find for the binding energy of $\sigma$ a linear dependence on the adsorption distance, in agreement with other calculations. ${ }^{43}$ For what concerns the linewidth of the $\sigma$ resonance we found for $\mathrm{Cs} / \mathrm{Cu}(111)$ an elastic contribution of $22 \mathrm{meV}$ that corresponds to a lifetime of $30 \mathrm{fs}$. This value is un upper limit for the experimental lifetime. Indeed, we consider only the elastic decay channel that is the most efficient on free electron metal surfaces and that can be directly related to the linewidth of the resonances in the DOS. Further inelastic processes can contribute to the lifetime, making the decay time shorter. ${ }^{67}$ Our elastic linewidth is slightly larger than that reported by Borisov et al., ${ }^{45}$ using the WPP method.

At the equilibrium adsorption distance the dispersion-less $\sigma$ resonance lies in the surface-projected energy gap at the $\bar{\Gamma}$ point, so the transfer of electrons is prevented, due to the absence of substrate states available for occupation. Nevertheless, the energy gap is not absolute but varies with $k_{\|}$so this blockade effect is only partial. Indeed, because of the coupling between different $k_{\|}$, the $\sigma$ resonance can hybridize also with states at larger $k_{\|}$, resulting in the nonzero linewidth observed in the DOS. Such hybridization is more efficient for states that are localized in space. Indeed, their Fourier transform includes relevant high $-k_{\|}$contributions that can hybridize with substrate states.

This interpretation is confirmed also by $a b$ initio calculations of Bormet et al. of adatoms on realistic $\mathrm{Al}(111){ }^{12}$ Their results show that on a free electron like surface, with an almost absent energy gap, the adatom induced resonances are wider, in agreement with the finding on jellium. In Fig. 2 are reported the DOS's for all the alkali adatoms adsorbed on the $\mathrm{Cu}(111)$ surface at their equilibrium distances. Our calculated elastic linewidths and binding energies for $\sigma$ are reported in Table I for all the alkali adatoms. The linewidth of the $\sigma$ resonance reduces passing from $\mathrm{K}$ to $\mathrm{Cs}$, according to the larger adsorption distance of heavy alkalis, which determines a 
smaller overlap with bulk states. The large value that we found for $\mathrm{Na} / \mathrm{Cu}(111)$ is probably due to an additional electronic state that contribute to this peak, as explained in the following section.

A second resonance appears in the unoccupied DOS of $\mathrm{Cs} / \mathrm{Cu}(111)$, at about $1 \mathrm{eV}$ below the vacuum level (vertical solid line in the figure). This broad feature contains different contributions that are accidentally almost coincident in energy in this system, while they are separated in the other alkali/ $\mathrm{Cu}(111)$ systems. The resonance has mainly $m=1$ and $m=2$ character. The $m=1$ contribution can be related to the $\pi$ resonance observed experimentally by AR-2PPE for

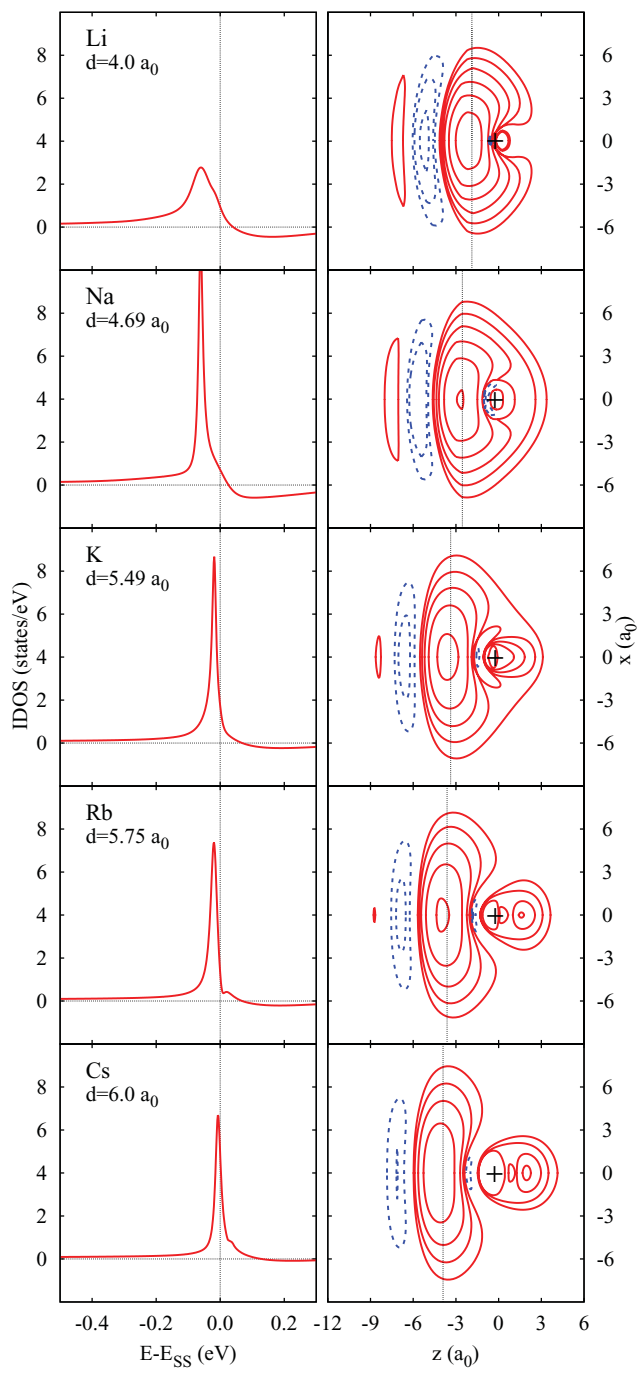

FIG. 3. (Color online) (Left panel) Occupied IDOS of al$\mathrm{kali} / \mathrm{Cu}(111)$ at the equilibrium adsorption distance. (Right panel) Induced charges at the equilibrium adsorption distances $(d)$. The origin of the axes corresponds to the adatom position. Red solid and blue dashed lines identify an increase and depletion of electronic charge, respectively. The starting electronic density for the contours is 0.0002 for both positive and negative values and a logarithmic contour spacing is used. The vertical line marks the image plane position. off-normal detection. ${ }^{64}$ In heavy alkalis a further $m=1$ peak ( $\pi^{\prime}$ in Fig. 3) can be found at higher energies. The $\pi$ and $\pi^{\prime}$ resonances are related to the $p$ and $d$ atomic levels, in particular to their component parallel to the surface. The $\pi$ resonance of $\mathrm{Cs} / \mathrm{Cu}(111)$ has a quite large linewidth, due to the partial hybridization with $\pi^{\prime}$. However, for $\mathrm{K}$ and $\mathrm{Rb}$ adatoms, where the energy separation between the $m=1$ peaks is larger, $\pi$ results narrower than the $\sigma$ resonance. Finally, in the adsorbed $\mathrm{Li}$ and $\mathrm{Na}$ the $\pi$ state is broadened by the hybridization with the upper bulk band.

Further wider $\sigma^{\prime}(m=0)$ and $\delta(m=2)$ contributions can be identified in the DOS of $\mathrm{K}, \mathrm{Rb}$, and Cs. They are related to the different $m$ components of the $d$ atomic level that are no more degenerate in the surface potential. The different linewidths of these resonances in the heavy alkali adsorbed systems is due to a different degree of hybridization with the substrate states which depends on many factors (symmetry, energy position, adsorption distance). The binding energy and linewidth of all the induced resonaces are summarized in Table I.

\section{DISCUSSION}

The DOS analyzed in the previous section for $\mathrm{Cs} / \mathrm{Cu}(111)$ shows a completely empty $\sigma$ resonance at the equilibrium adsorption distance. This result is in agreement with the experiments, confirming that the single valence electron of the alkali adatom is transferred to the substrate. Nevertheless, the binding energy of the $\sigma$ resonance is not sufficient to clarify the nature of the charge transfer, i.e., to characterize the adatom-surface bond. In this section we will analyze some aspects of the electronic properties that can elucidate this point, also comparing the behavior of different alkali adatoms.

\section{A. Occupied density of states and charge densities induced by the adatom}

We start our analysis of the adatom-surface interaction considering the changes induced in the occupied electronic states of the system on the adsorption of the alkali adatom. We refer to Fig. 3, which shows the induced density of states (IDOS) (left panel) and the associated induced charge densities $\left(\rho_{\text {ind }}\right)$ (right panel) for all the alkalis on $\mathrm{Cu}(111)$ at the equilibrium adsorption distance. The IDOS $\left(\rho_{\text {ind }}\right)$ is obtained subtracting the DOS (charge density) of the clean surface to that of the interacting system, both evaluated in the whole embedded region.

The IDOS's show the localization of the Shockley state (which is the reference for the energies), already described for $\mathrm{Cs} / \mathrm{Cu}(111)$. Note that this phenomenon is accompanied by a decrease of the DOS for energies slightly above the SS, due to a partial depletion of such a state.

We found that at the equilibrium adsorption distance the energy localization of the SS reduces along the period: the split off due to $\mathrm{Li}$, of the order of $50 \mathrm{meV}$, is about 6 times larger than that obtained for Cs. This is related to the lower adsorption distance of $\mathrm{Li}$ with respect to $\mathrm{Cs}$ and also to its larger attractive strength, due to the reduction of the ionization potential along a period. Indeed, the same trend is verified 
also considering all the adatoms at the same distance from the surface.

For what concerns the linewidth, the resonances induced by $\mathrm{Li}$ and $\mathrm{Na}$ are broader than those observed in the $\mathrm{K}, \mathrm{Rb}$, and Cs IDOS's, confirming the larger perturbative effect of the former. In addition, the line shape of the peak in the IDOS of light alkalis ( $\mathrm{Li}, \mathrm{Na}$ ) is asymmetric, suggesting that also another adatom induced electronic state could contribute to this resonance. In order to understand the possible origin of the shoulder on the right of the peak we consider the induced charge densities of the interacting systems.

Looking at the right panel of Fig. 3 one can note, for all the systems, an increase of charge density (red solid lines) in the region between the atom and the surface and a depletion of electrons (blue dashed lines) just inside the surface, where a modulation of the electronic charge can be observed. The electronic charge that spills out from the surface is mainly localized at the image plane, located at $2.1 \mathrm{a}_{0}$ from the surface (vertical line in the plot). This electronic cloud acts as a screening charge distribution, i.e., as the response of the system to the adsorption of the alkali adatom. In $\mathrm{Li}$ and $\mathrm{Na}$ the deeper penetration of the charge oscillations in the substrate explains the larger elastic width of the resonance in the IDOS, as due to a greater hybridization with bulk states. Light alkalis differ from the heavy ones also for what concerns the shape of the charge distribution. The induced electronic density partially surrounds the $\mathrm{Li}$ and $\mathrm{Na}$ adatoms. In $\mathrm{K}, \mathrm{Rb}$, and $\mathrm{Cs}$ it is mainly localized at the image plane and a charge polarization on the adatom is observed. These differences in $\rho_{\text {ind }}$, and the peculiarities observed in the IDOS's, suggest that Li and Na could induce the formation of an occupied bonding state. In the wake of such a hypothesis the adatom-surface bonding could have a partial covalent character for light alkalis while it would be mainly ionic for the heavy ones.

\section{B. Amplitude of the $\sigma$ resonance wave function}

In the hypothesis of a partially covalent bond, a bondingantibonding doublet should form. The atomic level involved in the covalent bond is the $s$ valence level, interacting with the $\mathrm{Cu}(111)$ states. In this case the $\sigma$ resonance should display a partial antibonding character for these systems. In Fig. 4 the squared modulus of the wave function of the $\sigma$ resonance induced by $\mathrm{Li}$ and $\mathrm{Cs}$ on $\mathrm{Cu}(111)$ is reported. The two limiting cases are considered: $\mathrm{Li}(\mathrm{Cs})$ is indeed expected to have the most covalent (ionic) character. In both systems the spherical character of the $s$ atomic level is perturbed by the interaction with the substrate. The higher wave function amplitude is found on the right of the atom (in the vacuum side) and this maximum appears more intense in $\mathrm{Li}$ than in $\mathrm{Cs}$ adatom.

The antibonding character of the resonance, i.e., a more intense amplitude of the wave function on the vacuum, can be deduced for Li more than for Cs, as expected.

In conclusion, the more directional character of the wave function in $\mathrm{Li}$, both for occupied and unoccupied states, and their spatial distribution with respect to the adatom, confirm the presence of a covalent contribution to the light alkalis/surface bond. The reason of such a difference can be found both in

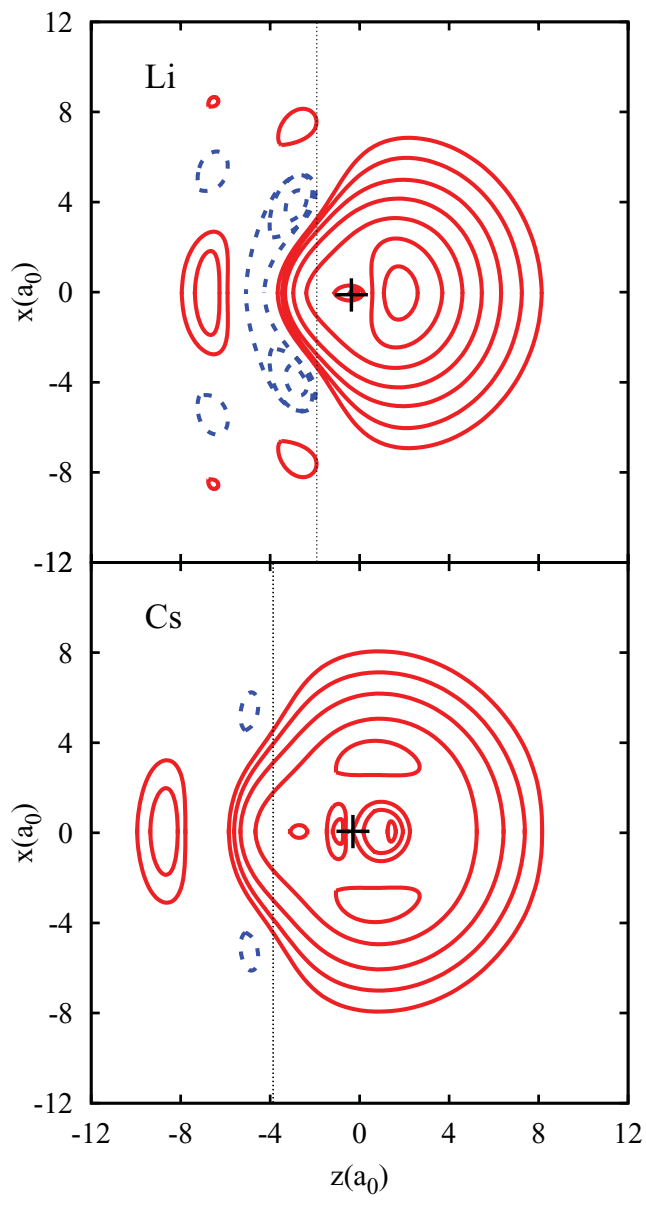

FIG. 4. (Color online) Variation of the squared amplitude of the $\sigma$ resonance induced by $\mathrm{Li}$ and $\mathrm{Cs}$ adatoms at the equilibrium adsorption distance. Red solid and blue dashed lines identify an increase and depletion of electronic charge, respectively. The starting electronic density for the contours is 0.0002 for both positive and negative values and a logarithmic contour spacing is used.

the lower adsorption distance of $\mathrm{Li}$ and $\mathrm{Na}$ and in their larger ionization potential that makes more difficult the complete transfer of the valence electron to the surface.

\section{Dipole moments and effective charge}

The partial transfer of the $s$ valence electron from the alkali atom to the substrate generates a surface dipole which is responsible for the local work function lowering. We calculate the dipole as

$$
\mu=\int d^{3} \mathbf{r} \rho_{\text {ind }}(\mathbf{r})\left(z-z_{\text {atom }}\right)+(1-Q)\left(z_{\text {im }}-z_{\text {atom }}\right),
$$

where

$$
Q=\int d^{3} \mathbf{r} \rho_{\text {ind }}(\mathbf{r}),
$$

i.e., the induced electronic charge in the embedded sphere. This quantity depends on the adsorption distance: We found 


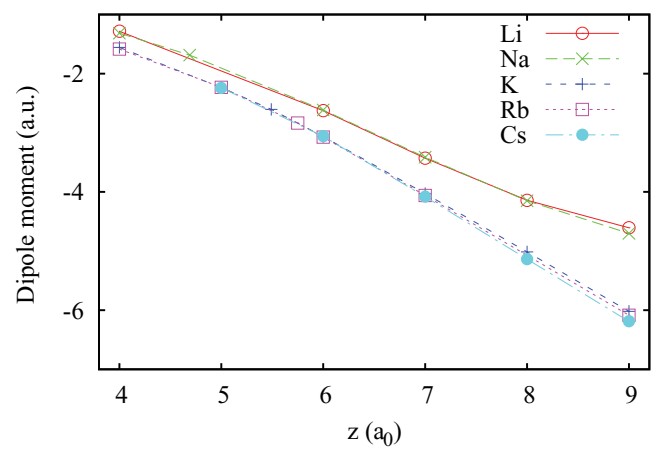

FIG. 5. (Color online) Dipole moments as a function of adatomsurface distance.

0.85 electrons in the sphere for $\mathrm{Li}$, which has the shortest equilibrium adsorption distance, while this value reduces to 0.72 electrons for Cs. The second term in Eq. (1) is a correction introduced to account for the effect of the electronic charge outside the embedded sphere, considering that one more valence electron is expected in the interacting system on the adsorption of the alkali atom. We assume that this small charge lies at the image plane.

In Fig. 5 the dipole moments as a function of the adatomsurface distance are reported for the series of alkali atoms. Two aspects can be noted: both the absolute value and the slope of $\mu(z)$ differ for light and heavy alkalis, suggesting a different interaction with the surface. The dipole variation with the atomic displacement is related to the effective charge $q^{*}$ on the adatom. ${ }^{68,69}$ This quantity is a measure of the response of the system to an external field. A completely ionized adatom is expected to behave in an electric field as a positive unitary charge $\left(q^{*}=1\right)$ while a partially neutralized ion retains an effective charge, $q^{*}<1$. The results in Fig. 5 show that, in proximity of the equilibrium adsorption distance of every alkali atom, $\mu$ is a nearly linear function of $z$. A linear fit of the data gives a $q^{*}$ equal to 0.7 and 1 electron for the light and heavy alkalis, respectively.

We can conclude that the adsorption of $\mathrm{Li}$ and $\mathrm{Na}$ induces a charge reorganization at the surface that partially neutralizes the positive charge atom. In other words, the light alkali adatoms are not completely ionized on such a surface.

Further evidence that supports this interpretation is the behavior of the induced charges at increasing adatom-surface distances that are reported in Fig. 6 for the two limiting cases of $\mathrm{Li}$ and $\mathrm{Cs}$. The electronic cloud follows the $\mathrm{Li}$ atom during the displacement from the surface. At large distances it appears considerably deformed, including a partially spherical charge distribution around the adatom and a localized screening charge near the image plane. For Cs, the charge distribution does not change considerably in shape, the screening charge remains localized in front of the surface, and the polarization of the alkali adatom gradually disappears. A similar analysis has been performed also for $\mathrm{Na}, \mathrm{K}$, and $\mathrm{Rb}$, confirming that $\mathrm{Na}$ behaves as $\mathrm{Li}$ and $\mathrm{K}$ and $\mathrm{Rb}$ as the Cs adatom. This evidence suggests that $\mathrm{Li}$ and $\mathrm{Na}$ partially retain their $s$ valence electron, confirming the partial covalent character of their interaction with the substrate.

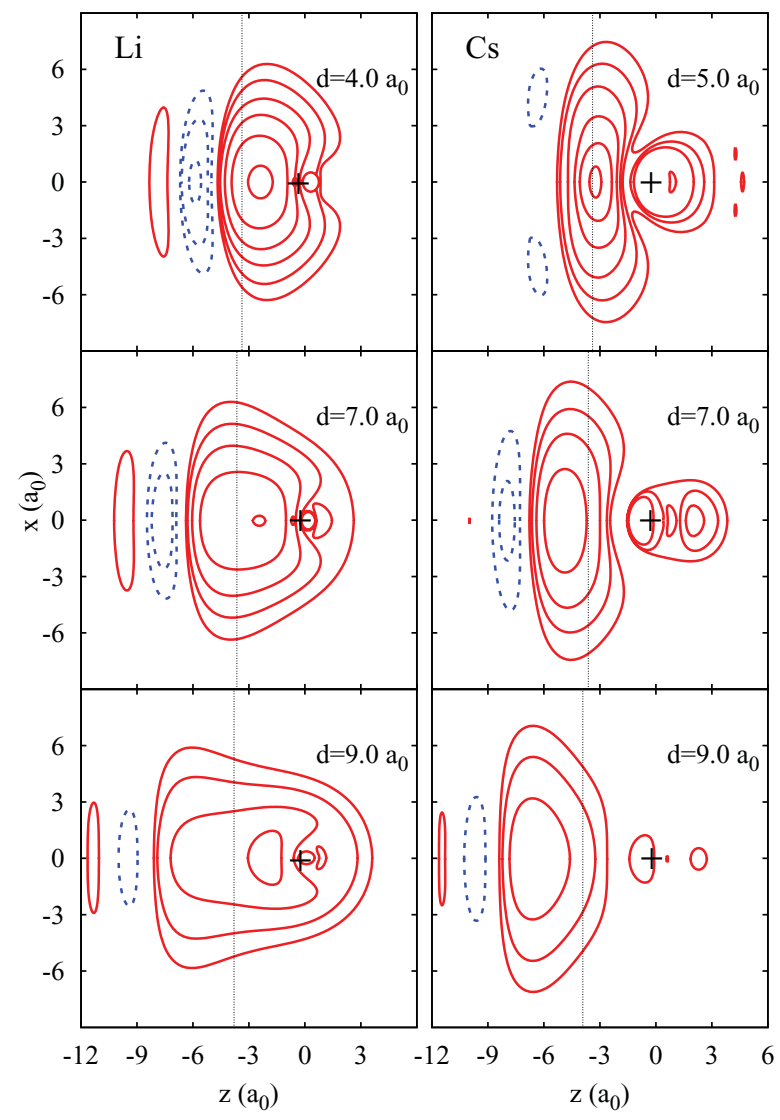

FIG. 6. (Color online) $\rho_{\text {ind }}$ of Li (left) and Cs (right) at increasing adatom-surface distance.

\section{CONCLUSIONS}

The analysis of the electronic properties of alkali adatom on the $\mathrm{Cu}(111)$ metal surface shows that, in order to describe the hybridization of the atomic states with the substrate ones and to analyze the aspects that contribute to their linewidth, the theoretical treatment has to account for some key aspects: (i) the infinite extension of the substrate that allows for a continuous spectrum, (ii) the presence of the projected band gap, (iii) a correct value of the work function of the substrate, (iv) a really isolated adsorbate, and (v) the presence of surface states.

Using our method, which satisfies all these requirements, we have characterized the alkali adatom/ $\mathrm{Cu}(111)$ system. The first relevant feature in the spectrum of the interacting system is the presence of a localized state just below the Shockley state of the pristine clean surface. This is due to the attractive alkali potential acting on the two-dimensional electronic state. The second relevant and conclusive result concerns the unoccupied resonances found in many experiments. Our $a b$ initio calculation shows a very satisfactory agreement in terms of binding energies and linewidth. Finally, on the basis of some differences in charge density distributions, dipole moments, effective charges we have concluded that $\mathrm{Li}$ and $\mathrm{Na}$ form a partially covalent bonding with the $\mathrm{Cu}(111)$ surface while $\mathrm{K}$, $\mathrm{Rb}$, and $\mathrm{Cs}$ bond with the substrate in a purely ionic fashion. 
${ }^{1}$ T. J. Killian, Phys. Rev. 27, 578 (1926).

${ }^{2}$ G. S. Tompa, M. Seidl, W. C. Ermler, and W. E. Carr, Surf. Sci. 185, L453 (1987)

${ }^{3}$ A. Hohlfeld, M. Sunjic, and K. Horn, J. Vac. Sci. Technol. A 5, 679 (1987).

${ }^{4}$ J. Paul, J. Vac. Sci. Technol. A 5, 664 (1987).

${ }^{5}$ D. Heskett, K.-H. Frank, E. E. Koch, and H. J. Freund, Phys. Rev. B 36, 1276 (1987).

${ }^{6}$ T. Kan, K. Mitsukawa, T. Ueyama, M. Takada, T. Yasue, and T. Koshikawa, Surf. Sci. 460, 214 (2000).

${ }^{7}$ E. M. Oelling and R. Miranda, Surf. Sci. 177, L947 (1986).

${ }^{8}$ G. Pacchioni and P. S. Bagus, Surf. Sci. 286, 317 (1993).

${ }^{9}$ H. Ishida, Phys. Rev. B 40, 1341 (1989).

${ }^{10}$ M. Scheffler, C. Droste, A. Fleszar, F. Máca, G. Wachutka, and G. Barzel, Physica B 172, 143 (1991).

${ }^{11}$ G. K. Wertheim, D. M. Riffe, and P. H. Citrin, Phys. Rev. B 49, 4834 (1994).

${ }^{12}$ J. Bormet, J. Neugebauer, and M. Scheffler, Phys. Rev. B 49, 17242 (1994).

${ }^{13}$ I. Langmuir and K. H. Kingdon, Science 57, 58 (1923).

${ }^{14}$ I. Langmuir and K. H. Kingdon, Proc. R. Soc. London A 107, 61 (1925).

${ }^{15}$ R. W. Gurney, Phys. Rev. 47, 479 (1935).

${ }^{16}$ A. J. Bennet and L. M. Falicov, Phys. Rev. 151, 512 (1966).

${ }^{17}$ J. P. Muscat and D. M. Newns, Surf. Sci. 74, 355 (1978).

${ }^{18}$ J. P. Muscat and D. M. Newns, Surf. Sci. 84, 262 (1979).

${ }^{19}$ J. W. Gadzuk, J. K. Hartman, and T. N. Rhodin, Phys. Rev. B 4, 241 (1971).

${ }^{20}$ T. B. Grimley, J. Phys. C: Solid State Phys. 3, 1934 (1970).

${ }^{21}$ P. Hohenberg and W. Kohn, Phys. Rev. 136, B864 (1964).

${ }^{22}$ N. D. Lang and A. R. Williams, Phys. Rev. B 18, 616 (1978).

${ }^{23}$ H. Ishida, Phys. Rev. B 38, 8006 (1988).

${ }^{24}$ I. Panas, J. Schüle, P. E. M. Siegbah, and U. Wahlgren, Chem. Phys. Lett. 149, 265 (1988).

${ }^{25}$ G. te Velde and E. J. Baerends, Chem. Phys. 177, 399 (1993).

${ }^{26}$ N. D. Lang, Solid State Comm. 9, 1015 (1971).

${ }^{27}$ N. D. Lang and W. Kohn, Phys. Rev. B 3, 1215 (1971).

${ }^{28}$ H. Ishida, Phys. Rev. B 42, 10899 (1990).

${ }^{29}$ G. Fratesi, G. Alexandrowicz, M. I. Trioni, G. P. Brivio, and W. Allison, Phys. Rev. B 77, 235444 (2008).

${ }^{30}$ E. V. Chulkov and V. M. Silkin, Surf. Sci. 215, 385 (1989)

${ }^{31}$ C. Corriol, V. M. Silkin, D. Sanchez-Portal, A. Arnau, E. V. Chulkov, P. M. Echenique, T. M. von Hofe, J. Kliewer, J. Kröger, and R. Berndt, Phys. Rev. Lett. 95, 176802 (2005).

${ }^{32}$ K. Niedfeldt, E. A. Carter, and P. Nordlander, Surf. Sci. 600, L291 (2006).

${ }^{33}$ T. B. Grimley and C. Pisani, J. Phys. C: Solid State Phys. 7, 2831 (1974).

${ }^{34}$ O. Gunnarson, H. Hjelmberg, and B. I. Lundqvist, Phys. Rev. Lett. 37, 292 (1976).

${ }^{35}$ N. Fischer, S. Shuppler, T. Fauster, and W. Steinmann, Surf. Sci. 314, 89 (1994).

${ }^{36}$ D. Tang and D. Heskett, Phys. Rev. B 47, 10695 (1993).

${ }^{37}$ D. A. Arena, F. G. Curti, and R. A. Bartynski, Phys. Rev. B 56, 15404 (1997).
${ }^{38}$ M. Bauer, S. Pawlik, and M. Aeschlimann, Phys. Rev. B 55, 10040 (1997).

${ }^{39}$ M. Bauer, S. Pawlik, R. Burgermeister, and M. Aeschlimann, Surf. Sci. 402, 62 (1998).

${ }^{40}$ M. Bauer, S. Pawlik, and M. Aeschlimann, Phys. Rev. B 60, 5016 (1999).

${ }^{41}$ S. Ogawa, H. Nagano, and H. Petek, Phys. Rev. Lett. 82, 1931 (1999).

${ }^{42}$ H. Petek, H. Nagano, M. J. Weida, and S. Ogawa, J. Phys. Chem. B 105, 6767 (2001).

${ }^{43}$ J. Zhao et al., Phys. Rev. B 78, 085419 (2008).

${ }^{44}$ J. W. Gadzuk, Phys. Rev. B 79, 073411 (2009).

${ }^{45}$ A. G. Borisov, J. P. Gauyacq, E. V. Chulkov, V. M. Silkin, and P. M. Echenique, Phys. Rev. B 65, 235434 (2002).

${ }^{46}$ S. Achilli, S. Caravati, and M. I. Trioni, Surf. Sci. 601, 4048 (2007).

${ }^{47}$ S. Achilli, S. Caravati, and M. I. Trioni, J. Phys. Condens. Matter 19, 305021 (2007).

${ }^{48}$ S. Achilli, M. I. Trioni, E. V. Chulkov, P. M. Echenique, V. Sametoglu, N. Pontius, A. Winkelmann, A. Kubo, J. Zhao, and H. Petek, Phys. Rev. B 80, 245419 (2009).

${ }^{49}$ M. I. Trioni, G. P. Brivio, S. Crampin, and J. E. Inglesfield, Phys. Rev. B 53, 8052 (1996).

${ }^{50}$ E. V. Chulkov, V. M. Silkin, and P. M. Echenique, Surf. Sci. 437, 330 (1999).

${ }^{51}$ O. Gunnarson and H. Hjelmberg, Phys. Scripta 11, 97 (1975).

${ }^{52}$ J. E. Inglesfield, J. Phys. C: Solid State Phys. 14, 3795 (1981).

${ }^{53}$ J. E. Inglesfield, e-print arXiv:1003.2282 (2010).

${ }^{54}$ B. Simon, Ann. Phys. (NY) 97, 279 (1976).

${ }^{55}$ F. E. Olsson, M. Persson, A. G. Borisov, J.-P. Gauyacq, J. Lagoute, and S. Fölsch, Phys. Rev. Lett. 93, 206803 (2004).

${ }^{56}$ L. Limot, E. Pehlke, J. Kröger, and R. Berndt, Phys. Rev. Lett. 94, 036805 (2005).

${ }^{57}$ B. Lazarovits, L. Szunyogh, and P. Weinberger, Phys. Rev. B 73, 045430 (2006).

${ }^{58}$ S. Lounis, P. Mavropoulos, P. H. Dederichs, and S. Blügel, Phys. Rev. B 73, 195421 (2006).

${ }^{59}$ J. P. Gauyacq, A. G. Borisov, and A. K. Kazansky, Appl. Phys. A 78, 141 (2004).

${ }^{60}$ W. Jacob, E. Bertel, and V. Dose, Phys. Rev. B 35, 5910 (1987).

${ }^{61}$ H. B. Nielsen and W. Thowladda, Surf. Sci. Lett. 284, L426 (1993).

${ }^{62}$ H. Petek, M. J. Weida, H. Nagano, and S. Ogawa, Science 288, 1402 (2000).

${ }^{63}$ D. Kröner, S. Klinkusch, and T. Klamroth, Surf. Sci. 602, 3148 (2008).

${ }^{64}$ A. G. Borisov et al., Phys. Rev. Lett. 101, 266801 (2008).

${ }^{65}$ A. G. Borisov, A. K. Kazansky, and J. P. Gauyacq, Surf. Sci. 430, 165 (1999).

${ }^{66}$ K. Niedfeldt, E. A. Carter, and P. Nordlander, J. Chem. Phys. 121, 3751 (2004).

${ }^{67}$ J. P. Gauyacq, A. G. Borisov, and M. Bauer, Progr. Surf. Sci. 82, 244 (2007).

${ }^{68}$ E. A. Colbourn and J. E. Inglesfield, Phys. Rev. Lett. 66, 2006 (1991).

${ }^{69}$ S. Clarke, J. E. Inglesfield, M. Nekovee, and P. K. de Boer, Phys. Rev. Lett. 80, 3571 (1998). 